\title{
Perception and Behavioral Intention of Cycling Space on Urban Greenway
}

\author{
Feng Qinhui $^{1}$, Wang Weiqiang ${ }^{1}$, Allam Maalla ${ }^{1 *}$ \\ ${ }^{1}$ Guangzhou College of Commerce, Guangzhou, China, 511363
}

\begin{abstract}
To explore the spatial power and spatial relationship of urban greenway sports cultural memory, using literature data induction analysis method, questionnaire survey method, interview method, and other research methods, the connotation of urban greenway sports cultural memory is analyzed, and the urban greenway sports are explained. Using Lefebvre's ternary dialectics to study the production process of Guangzhou greenway's sports cultural memory space, its spatial practice is in the interpretation of Guangzhou's Greenway sports cultural memory. The government is the leading force in the memory of greenway sports culture. It is a necessary prerequisite for the cultural memory of greenway sports, and the public is the main body of the cultural memory of greenway sports. The spatial representation is in the context of the cultural memory of greenway sports, sorting out the hard memory and soft memory in the memory field of Guangzhou Greenway Sports Culture. The representational space is the sports cultural memory space experienced by the greenway activists, space directly "lived" by the greenway activists, and the internalization of the cognition, experience, and spatial representation of the sport's cultural memory of the greenway activists.
\end{abstract}

\section{Introduction}

"14th Five-Year Plan" is committed to the new development concept of innovation, openness, greenness, and sharing, and achieves the goal of people's growing demand for a better life. As an important carrier for the happiness of recreational people, greenways are not only a project to promote the construction of ecological civilization, but also an ideal place for residents to develop leisure and tourism [1]. As a product of the lowcarbon culture in the new era, Greenway Sports has become a benchmark for happiness in Guangdong, improvement of people's quality of life, and fitness practice [2]. Every time a new bicycle lane is built in Copenhagen, Denmark, the number of cyclists will increase by $20 \%$, and the number of drivers will decrease by $10 \%[3]$. A big data study of my country Bicycle Association shows that $71 \%$ of cyclists have a college degree, and the core age is between 18-24 years old [4]. College students have a strong environmental awareness, and college cycling associations have developed rapidly. Greenway cycling for college students has become a fashion, and it has gradually become the main group of urban greenway cycling. While demonstrating the relationship between the spatial environment, functional system, and supporting services of Guangdong Greenway on the riding behavior of college students, this research also provides effective spatial distribution planning services for the complex greenway environment and provides an in-depth understanding of Guangdong
Greenway Sports Public The practice profile of the service provides feasible and effective investigation methods.

\section{Research Design}

\subsection{Overview of the study area}

This study selects the greenway riding behavior of universities in Guangdong as the research object, because Guangdong Province is the first to propose greenway construction, and relying on greenway construction to share happiness, Guangdong, under the premise of vigorously promoting the construction of greenway supporting facilities, take fitness, sports, and leisure as the research object. The main function of the greenway sports public service came into being. At the end of December 2018, Guangdong province built a continuous and highly feasible $18019 \mathrm{~km}$ complete greenway network to provide a carrier of fitness, leisure, sightseeing, and commuting for greenway cycling [19]. The Guangdong Pearl River Delta Greenway has developed a unique Guangdong-style and domestically leading urban greenway sports and fitness demonstration site, connecting the three metropolitan groups of Guangfo Zhao, Shenzhen, Guanhua, and Zhuzhongjiang, greatly enriching cycling resources. Cycling has become a fashion, providing a wealth of important outdoor sports opportunities for young people to travel between cities. The ecological, low-carbon, cultural, and economic functions of

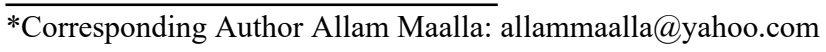


Guangdong Greenway are coupled with Secretary Xi Jinping's five development concepts. In terms of greenway sports and leisure functions that meet the needs of people from different classes and different regions, the study of greenway sports is the most popular among Guangdong college students. Representative and typical.

\subsection{Factor analysis results}

Through the factor analysis of the formal questionnaire, extracting 5 factors with a characteristic value greater than 1 can explain $72.650 \%$ of the amount. And named these five factors, namely: accessibility perception, service perception, supporting perception, environmental spatial characteristics, and riding behavior intention. Fig. 1 and Fig. 2 show the factor loading and variance explanation of each dimension and list the variables with higher coefficient loading in each coefficient. The 36 observation variables used in the survey have factor loads greater than 0.5 , which indicates that the observed variables have achieved the purpose of this research.

\subsection{Questionnaire survey}

This study issued a formal questionnaire in November 2020. The subjects of the survey were the cycling groups in Guangdong universities. The survey selected 6 cities in the Pearl River Delta (Guangzhou, Shenzhen, Dongguan, Zhuhai, Jiangmen, Zhongshan); 2 cities in eastern Guangdong (Shantou, Shantou, Questionnaires were distributed by groups of 12 college cycling associations in 2 cities in western Guangdong (Zhanjiang and Maoming) and 2 cities in northern Guangdong (Shaoguan, Heyuan). The questionnaires were mainly electronic, to improve the filling rate of the questionnaires. Through the relationship between the instructors of these college cycling associations, the questionnaires were sent in the form of red envelopes and thank-you letters. A total of 461 questionnaires were recovered, of which 379 valid questionnaires. Invalid questionnaires with the same scores for more than 10 consecutive items have been eliminated. The effective questionnaire recovery rate reached $82.2 \%$.

\section{Descriptive analysis of the questionnaire}

\subsection{Attribute Characteristics of the Sample}

It can be seen from Fig. 1 that in the descriptive statistics of the survey sample, the gender distribution of the sample is relatively reasonable. Females in the greenway cycling group in colleges and universities are slightly higher than men, and the liberal arts majors are slightly higher than the science majors. $65 \%[12]$, it may be that there are more liberal arts colleges than science colleges in the survey, or it may be that to obtain a better riding environment and improve the safety of riding, more girls choose to join the cycling association. Most of the universities studied are concentrated in the Pearl River Delta (48.6\%). The cities in the Pearl River Delta have developed economies and mature green roads, which are the gathering places for universities in Guangdong. Most of the investigators' grades are concentrated in sophomores and juniors, accounting for $79.3 \%$ of the overall sample. During this period, as students become familiar with the urban greenways around the school, their willingness to buy bicycles has increased, and greenway riding and leisure behavior increase. The freshman has a fresh sense of cycling, and the urgency of being familiar with the urban greenway environment makes the freshmen have a strong tendency to join the greenway cycling group. The number of samples in the senior year is small, mainly after four years of university life and cycling, they are familiar with the greenway environment of the city where they are located and need to go out for internships and work frequently and spend less time on urban greenway leisure.

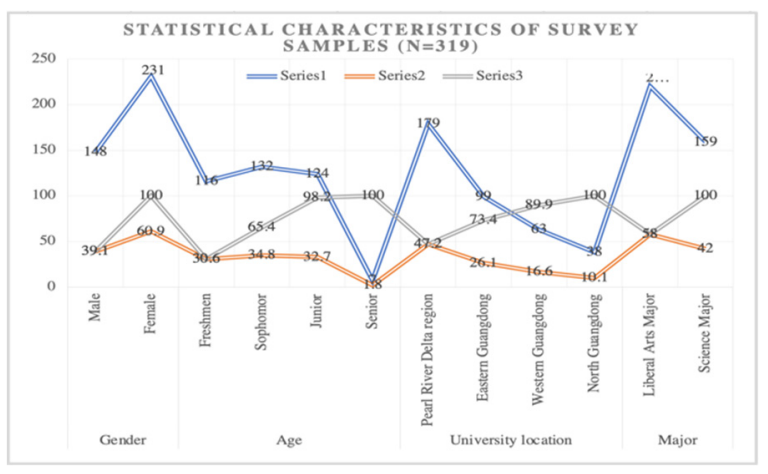

Fig. 1. Statistical Characteristics of Survey Samples $(\mathrm{N}=319)$

\subsection{Descriptive analysis of the scale}

The statistical results in Fig.2 show that, among the constituent factors of cycling space perception, the perception of cycling matching perception $(\mathrm{M}=3.41)$ and service perception $(\mathrm{M}=3.40)$ is relatively low, and Guangdong college cycling groups need to visit in person To effectively perceive the cycling facilities and services of urban greenways, some cycling groups may only perceive the high-quality spatial environment of Guangdong greenway riding $(\mathrm{M}=3.61)$. Riding my bicycle is only for relaxation, without personal experience of urban greenways. Matching. As for the perception of accessibility of urban greenways $(M=3.49)$, on the one hand, the distance between the cycling groups to enter the greenway is relatively long, and on the other hand, the distance between the urban greenways in Guangdong is relatively long, and the college cycling groups cannot influence the whole city. Tao space environment for a complete experience. Greenway riding in Guangdong colleges and universities requires the organization and guidance of the association. As a group of young people, college students are the most important factor in the perception of riding space, followed by consideration of the accessibility of greenway riding, etc. factor. 


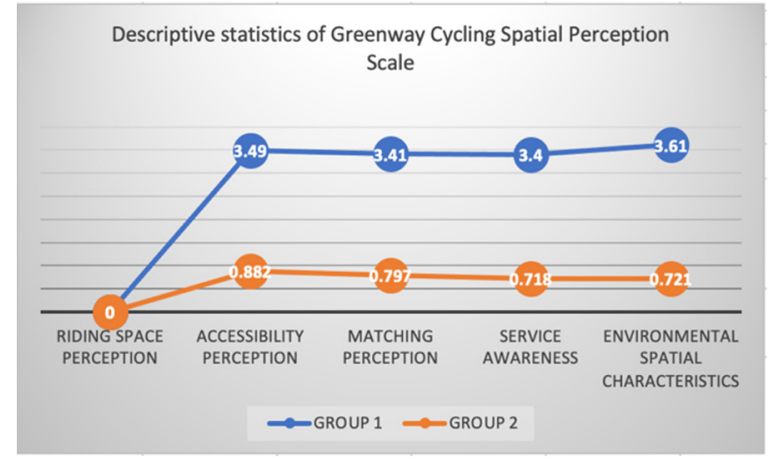

Fig. 2. Descriptive statistics of Greenway Cycling Spatial Perception Scale

\section{Discussion}

\subsection{Perceive the Internal Structure of The Greenway Cycling Space}

Aiming at the spatial characteristics of urban entertainment venues, from the perspective of behavioral cognition, we construct spatial perception attributes including five aspects: location perception, distance perception, functional integration perception, information field perception, and cultural-ecological perception [20]. In the urban greenway sports public entertainment space, except for the non-alone cycling group under the age of 10 , the largest proportion of the cycling group is the youth group[12], the height of the perception dimension of college students' cycling behavior and urban entertainment and leisure space Related, the accessibility perception (Q1-Q3) constructed after the factor molecule reflects the distance perception of Guangdong college students to reach the urban greenway. The accessibility of Guangdong greenways has been greatly improved. Studies have shown that Guangdong has formed a community greenway that can be reached when you go out, a community greenway can be reached in 5 minutes, and a provincial greenway can be reached in 30-45 minutes. Up to 4 levels of urban greenway sports and leisure system [20]. For example, Shenzhen University can reach the urban greenway when you go out, and 15 minutes to the urban area greenway-Shenzhen Bay Greenway; supporting perception space (Q4-Q7) represents the cycling fitness facilities provided by Guangdong Greenway, domestic greenway Sports facilities are developing towards automation and intelligence, which can meet the actual needs of college students for riding. For example, the shared bicycles and automatic vending machines in the greenway station are catering to the riding needs of college students; service perception (Q8- Q11) Represents the level of cycling services provided by Guangdong Greenway, for example: Xiti Inn in Zengcheng provides greenway cycling services for 15 yuan a day, which is in line with the consumption level of college students; environmental spatial characteristics (Q12-Q21) Guangdong Greenway The public space and landscape provided in the city increase the attractiveness of cycling groups, such as the cultural characteristic green roads in the hometown of overseas
Chinese in Jiangmen, Guangdong and the series of tower villages, meeting the needs of college students for cycling tourism to enjoy the world cultural heritage, and the increase in Zengcheng, Guangzhou Jiang Gallery Water Greenway meets the cycling and exercise needs of college students in scenic areas; cycling behavior intention (Q22Q36) represents the urban greenway cycling space in Guangdong to stimulate the enthusiasm and ability of cycling groups to exercise. With the urban greenway space, The gravitational pull of college students' physical exercise is constantly increasing. The greenway riding of college students takes into account the landscape and differences of the greenway, and follows the ecological pattern of "the harmony between man and nature". It is a desire and returns to nature in urban life. An important part of the city's cultural memory. The supporting facilities of urban greenways are certainly important, but accessibility, service level, and environmental spatial characteristics have become important indicators that affect the cycling activities of college students. Research on the spatial perception of urban greenway's internal structure and its mechanism of action shows that the supporting facilities, services, and environmental landscape of urban greenways are the driving force for greenway riding. This view is supported by previous research results: Improving the sports of urban greenways Spatial perception is an effective strategy to improve their sports behavior [17]. This has an inspiring effect on college students' greenway riding attempts, low-carbon travel, fashionable life, and the creation of a harmonious greenway sports culture atmosphere.

\subsection{Greenway cycling spatial perception and behavior intention}

The chronic network space of urban bicycle riding is characterized by multi-level and high mobility, and its behavior characteristics are closely connected with the environment, and there are many central nodes, which are consistent with the urban economic level distribution [19]. Urban cycling realizes people's cycling behavior and perception of greenway sports space. Greenway space environment is an important intermediary factor. Behavioral decision-making, in reality, is bounded and rational. It is easier to filter out other factors when perceiving the environment and being satisfied [twentythree]. People's subjective behavior decision-making originates from internal driving factors but is also affected by external physical space and environmental perception. Correlation analysis confirms that there is a correlation between greenway cycling space perception and behavior intention. Accessibility perception is positively correlated with cycling behavior intention $(\mathrm{r}=0.670)$. The distance between urban greenways and colleges is short, and the openness is good. The scale of greenway cycling can effectively promote the cycling group of Guangdong colleges and universities. Behavior, for example, students in Guangzhou University Town can reach the urban greenway when they go out, and they can reach the bioisland greenway in 5 minutes by cycling. The accessibility is strong, and the greenway for college students is large. 
The perception of supporting facilities is positively correlated with the intention of riding behavior $(\mathrm{r}=0.680)$. The supporting facilities for greenway riding are not only bicycle rental services, but also the guide signs for greenway riding, whether the road is level, the continuity of the greenway, and the connection Whether it is comfortable or not has a good role in promoting the riding behavior of college students in Guangdong. Because the perception of greenway service is related to the intention of greenway riding behavior $(\mathrm{r}=0.745)$, the quality of sports service and physical fitness guidance in the urban greenway space is improved. Can promote the riding behavior of college students. It is not difficult to find out through field investigations of Guangdong urban greenway sports supporting facilities that the greenway cycling paths in the new urban area are spacious. For example, the newly-built greenways in Guangzhou Knowledge City only have slow-moving paths over 4 meters, but the greenway connections have a large gap., And some drop more than $10 \mathrm{~cm}$, resulting in a very bad riding experience. There is a positive correlation between the environmental characteristics of the greenway space and the greenway riding behavior intention $(\mathrm{r}=0.835)$, and the attraction of the environmental space cannot be ignored. For example, the Huguangyan Scenic Spot in Zhanjiang attracts many nearby college students to ride around the lake on weekends, and Chaozhou's Hanjiang Greenway Scenic Area is also a choice for college students to ride. In addition to the greenway scenic area, greenway vegetation, greenway quality, greenway flatness, greenway rest facilities, greenway sanitation facilities, and greenway safety have the greatest influence on the riding behavior of Guangdong college students. Therefore, spatial perception is the internal driving force of cycling behavior intention and the most important factor in promoting greenway cycling among college cycling groups. The viewpoints of this research have certain practical significance. Meeting the psychological needs of urban greenway riding space can directly affect the behavior intention of college students in greenway riding. It is true that from the current urban cycling activities, students riding on behalf of the young force triggers urban shared bicycles to become a major urban problem. Guangdong's urban greenway bicycle system is mainly based on recreational cycling lanes and commuter lanes. During weekends, urban greenway cycling will experience "vehicle jams". The greenway channels available for cycling are relatively narrow and the greenway is slow. Sports space planning has not been resolved for a long time, which will have a certain impact on urban greenway cycling [20]. Even in the face of the more attractive cultural landscape of the old city, the interest and enthusiasm for greenway cycling will decline. Based on this, the greenway cycling space perception indirectly affects college students' greenway cycling investment, focusing on the humanized design and reasonable planning of the slow-moving greenway space, and connecting the city's various scenic spots and cultures in the form of a greenway as a "necklace" Legacy, to give young people more greenway riding options, make up for the unfavorable factors of urban greenway, promote college students to have a higher level of low-carbon and fashion awareness of greenway riding, and help college students get better greenway riding Behavior intention, enjoy the fun of riding on urban greenways.

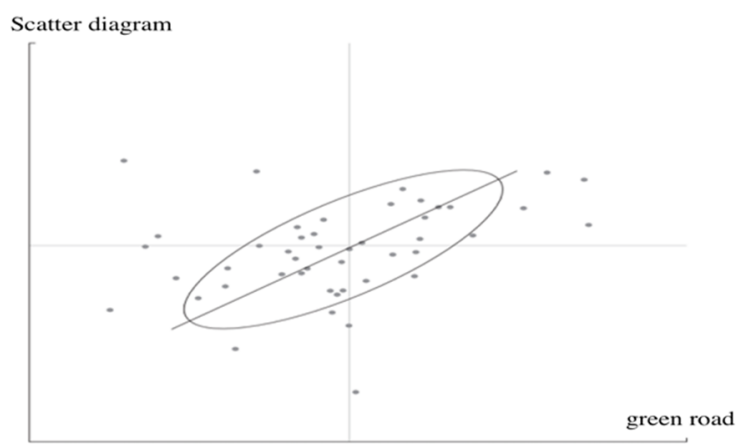

Fig. 3. Scatter diagram of four dimensions of green road riding behavior intention and spatial perception

\subsection{The relationship between principal component factors and overall satisfaction with cycling behavior intention}

Through regression analysis, the four principal component factors of greenway cycling space perception (accessibility perception, supporting perception, service perception, and environmental spatial characteristics) have a linear relationship with cycling behavior intention and have a significant relationship with greenway cycling behavior intention The order of sexual influence is environmental spatial characteristics, service perception, and accessibility perception. The spatial characteristics of the environment have become the primary factor influencing the greenway behavior intention of the cycling group in Guangdong colleges and universities. With the independent variable unchanged, the increase of the cycling behavior intention is 0.603 units, which is similar to that of Guangdong Urban Greenway Sports. The environmental benefits complement each other and are in line with the development of urban greenway sports public spaces. The formed Guangdong urban greenway cycling space network meets the greenway travel needs of college cycling groups. Urban greenways have become the first choice for landscape recreation and cycling fitness. , To meet the natural needs of young people to love and touch nature. In addition, with the independent variables unchanged, there are 0.191 service perception units and 0.123 accessibility perception units in the improvement of cycling behavior intention.

\section{Conclusion}

The deficiencies of this research still need follow-up improvement. Firstly, the perceptual guidance, emotional stimulation, cycling benefits and behavior preferences involved in behavioral intentions should be further tested; secondly, the research objects of Guangdong urban greenway cycling should be certain differences in the perception and behavioral intentions of urban greenway cycling among college students and other groups of people. There is a positive correlation between the environmental characteristics of the greenway space and 
the greenway riding behavior intention $(\mathrm{r}=0.835)$, and the attraction of the environmental space cannot be ignored. Finally, it is explored that the external driving force to produce the memory space of Guangzhou Greenway sports culture is government power, and the internal driving force is the integration and continuation of greenway cultural resources and the pursuit of individual meanings.

\section{ACKNOWLEDGMENTS}

This research was financially supported by Guangdong Provincial Department of Education's Young Innovative Talents Project (Humanities and Social Sciences) Project name: "Strategies for improving physical fitness based on the physical fitness test of college students-Taking Guangzhou Business College as an example"; Project approval number: 2020WQNCX078, And Guangdong Province Project, Construction of Teaching Team in Guangdong Province's Colleges and Universities Project "E-commerce Teaching Team" , (Project No. 2019SJJXTD01), And 2020 Guangdong Province Teaching Quality and Teaching Reform Project, Characteristic Majors, Data Science and Big Data Technology (Project No. 2020JTSZY01). And 2019 Guangzhou College of commerce Teaching Quality and Teaching Reform Project, Characteristic Majors, Data Science and Big Data Technology (Project No. 2019XJTSZY02). Corresponding Author Allam Maalla.

\section{References}

1. A. Maalla, "Research on DC Transmission Operation System Based on Analyze the Principle of Udi0," 2019 IEEE 4th Advanced Information Technology, Electronic, and Automation Control Conference (IAEAC), Chengdu, China, 2019, pp. 2580-2583, DOI: 10.1109/IAEAC47372.2019.8997870

2. $\mathrm{Li}$ Guoyu. Greenway Sports and Happiness Guangdong [J. Journal of Physical Education, 2013(2): 39-43.

3. Qiu Jian. Exploration of urban commuting greenway design based on commuting travel behavior [D]. Ya'an: Sichuan Agricultural University.

4. A. Maalla, "Development Prospect and Application Feasibility Analysis of Robotic Process Automation," 2019 IEEE 4th Advanced Information Technology, Electronic and Automation Control Conference (IAEAC), Chengdu, China, 2019, pp. 2714-2717, DOI: 10.1109/IAEAC47372.2019.8997983.

5. 2017 China Cycling Sports Big Data Report. [EB/OL]. [2018-05-06]. http://www.imxingzhe.com/document/344/,2018-56.

6. A. Maalla, "Current Status of Valve Base Electronics Equipment in DC Transmission System," 2020 IEEE 5th Information Technology and Mechatronics Engineering Conference (ITOEC), Chongqing,
China, 2020, pp. 1809-1812, doi: 10.1109/ITOEC49072.2020.9141831.

7. Fabos J G. Introduction and overview: the greenway movement, uses, and potentials of greenways[J]. Landscape and Urban Planning, 1995, 33(1-3): 1-13.

8. Siderelis C, Moore R. Outdoor Recreation Net Benefits of Rail-trails[J]. Journal of Leisure Research, 1995, 27(4):344-359.

9. East Coast Greenway Alliance. About the alliance . [EB/OL]. [2013-04-25]. http://www.greenway.org

10. Deenihan G, Caulfield B, O'Dwyer D. Measuring the Success of the Great Western Greenway in Irelands[J]. Tourism Management Perspectives, 2013, 7: 73-82.

11. Lumsdon L, Downward P, Cope A. Monitoring of Cycle Tourism on Long Distance Trails: The North Sea Cycle Route[J]. Journal of Transport Geography, 2004, 12(1): 13-22.

12. Lu Xutao, Hong Pengfei. Research on Cycling Behavior and Spatial Features Based on Web Text Analysis [J]. Journal of Beijing Sport University, 2018(5): 32-38.

13. Yu Yong, Tian Jinxia. A study on the relationship between riders' leisure involvement, leisure benefits, and happiness structure: Taking Zhaoqing Xinghu Bicycle Greenway as an example[J]. Tourism Tribune, 2013(2): 67-76.

14. Yang Shu, Zhang Weiya. A study on the correlation between the perceived image of the scenic spot and the satisfaction of recreation: Taking the cycling recreationists on the Zijin Mountain Greenway as an example [J]. Resource Development and Market, 2014 (10): 1268-1273.

15. Hoffman DD, Singh M, Prakash C. The Interface Theory of Perception[J]. Psychonomic Bulletin \& Review, 2015(6): 1-27.

16. PRUYT E, ISLAM T. On Generating and Exploring the Behavior Space of Complex Models[J]. System Dynamics Review, 2016, 31(4): 220-249.

17. Cai Yujun, Zhou Peng, et al. The relationship between urban residents' perception of public sports space and sports activities[J]. Journal of Chengdu Sport University, 2018(4): 48-53.

18. A. Maalla, "Current Status of Valve Base Electronics Equipment in DC Transmission System," 2020 IEEE 5th Information Technology and Mechatronics Engineering Conference (ITOEC), Chongqing, China, 2020, pp. 1809-1812, doi: 10.1109/ITOEC49072.2020.9141831.

19. Xie Dongxing. Research topics, techniques, and perspectives of greenway sports in my country in the new era [J]. Journal of Physical Education, 2019(2): 1-6.

20. Sports Bureau of Guangdong Province: Situation of Greenways in Guangdong Province. [EB/OL]. [20205-6]. http://tyj.gd.gov.cn/bigdata_qzty/content/post_2988 704.html 